\title{
Clippers: A Case which does not Respond to Steroid Treatment
}

\author{
Asuman $A^{1}$, Cemile $H^{1}$, Gümüştaş $\mathrm{AU}^{2}$, Gümüștaş $0^{3 *}$, Hakyemez $\mathrm{B}^{4}$ \\ and Ömer FT5 \\ ${ }^{1}$ YuksekIhtisas Training and Research Hospital Neurology Clinic, Turkey \\ 2,3Burtom Radiological Diagnostic Center, Turkey \\ ${ }^{4}$ Uludağ University Medical Faculty Radiology Department, Turkey \\ 5Uludağ University Medical Faculty Neurology Department, Turkey
}

Case Report

Volume 2 Issue 3

Received Date: August 28, 2018

Published Date: September 24, 2018

*Corresponding author: Oğuzhan Gümüştaş, Burtom Radiological Diagnostic Center, Department Of Radiology Burtom, Turkey, Tel: 05358380650; Email: oggugu@mynet.com

\begin{abstract}
In this study, we aimed to increase our knowledge by comparing clinical and brain magnetic resonance imaging (MRI) findings in CLIPPERS syndrome. A 34-year-old male patient was treated with a diagnosis of upper respiratory tract infections at centers where he had begun slowly with no previous history of illness and drug use, and had complaints of exacerbation, vomiting, anorexia and unbalanced gait. But the clinical features were not stable, visual impairment, speech slowing, swallowing and chewing difficulties were added, neurological evaluation was performed, and high-dose pulse methylprednisolone therapy following brain MR was administered $1 \mathrm{~g} /$ day intravenously for five days. Oral methylprednisolone treatment was continued. Corticotherapy was partially responded and the patient had an aspiration pneumonia after three months and died at the fourth month of the illness. 1.5 tesla brain MRI was performed and T1w,T2w,T2 flair and diffuse axial sections were taken. Cerebrospinal fluid analysis was performed.

Brain MRI revealed diffuse hyperintense lesions at the level of pons and bilateral cerebellar hemispheres. There were slightly contrast enhancement. In the deep gray matter nuclei (basal ganglia and thalamus) and periventricular and subcortical there were also extensive punctate lesions observed in the fields. Brain MRI features were found to be compatible with CLIPPERS. Multiple sclerosis and other demyelinating diseases should be considered in differential diagnosis. But it was questioned whether or not the patient was responding to the steroid, and he was held responsible fort the late treatment. Early recognition may be a critical factor in stopping the deterioration of the clinic and positively affecting prognosis.

Keywords: Clippers; Multiple Sclerosis; Demyelinating Diseases
\end{abstract}

Abbreviations: MRI: Magnetic Resonance Imaging; CSF: Cerebrospinal Fluid; BBE: Bickerstaff's Brainstem
Encephalitis; CT: Computed Tomography; CNS: Central Nervous System. 


\section{Clinical Radiology \& Imaging Journal}

\section{Introduction}

CLIPPERS (Chronic lymphocytic inflammation with pontine perivascular enhancement responsive to steroids) was first defined in 2010 by Pittock, et al. and is the only form of pons-centered brainstem encephalitis. However, the causes and pathogenesis of CLIPPERS have not been fully understood to date. The main clinical findings were initially diplopia, gait ataxia and facial paresthesia. Nystagmus, dysarthria, dysphagia, and other findings may also occur when the disease progresses $[1,2]$. Clinical characteristics can be heterogeneous, they are versatile and variable, depending on the individual. There are significantly considerable differences about the age of onset. Age of onset varies between 13 and 86 years. It affects both genders.

The brain magnetic resonance (MR) imaging findings in CLIPPERS are very striking and have characteristic features, and there are significant similarities between the affected individuals. The most important feature of brain MR is the observation of lesions that enhance gadolinium in the form of numerous spotted or patched points and/or curves. Lesions extending to pons, cerebellar peduncles and cerebellum-spreading may also develop in adjacent central nervous system structures. It can spread to medulla oblongata or cervicothoracic spinal cord from caudal and to "midbrain" from anterior and even to the supratentorial regions such as cerebral white matter, corpus callosum, basal ganglia, capsula interna and thalamus. Lesions are typically smaller and fewer in number away from the pons, but increase in pons. Generally, they do not cause mass effect and minimal vasogenic edema can be seen $[3,4]$.

CLIPPERS diagnosis is based on clinical, radiological, laboratory and cerebrospinal fluid (CSF) studies and, if necessary, brain biopsy. Until now, diagnostic criteria for CLIPPERS have not been established. Extensive research is needed to exclude alternative situations that may mimic the CLIPPERS syndrome.

\section{Case}

A 34-year-old male patient complained of headache and insomnia lasting for a month with no previous illness or medication use history. At that time, however, he did not present to a hospital. When headache moderately continues, dizziness which starts mildly and gets severe over time, vomiting, loss of appetite and unbalanced walking complaints were added to clinical presentation. At the centers he applied, he was treated with "upper respiratory tract infection, sinusitis" and diagnoses. However, neurological evaluation was performed when not being able to stand, visual impairment, slowing of speech, swallowing and chewing difficulties were added to the clinical features and following brain MR result, he received high dose methyl prednisolone treatment as $1 \mathrm{gr} /$ day intravenously for 10 days. While maintenance treatment with oral methyl prednisolone was continued, horizontal nystagmus and rightward outward visual limitation, left peripheral facial paralysis, decreased bilateral "gag" reflex, dysphagia and dysphonia, deterioration in cerebellar tests, axial hypotonia, hypotonic tetraparesis and reflex abolish were detected. Examinations performed and results are shown in the Table 1. Despite corticotherapy, the patient's hypotonic tetraparesis, dysphagia, dysphonia, dysarthria were not recovered, he could not feed orally and become beddependent. Four months after the onset of the clinic, aspiration pneumonia has developed and our patient passed away. 1.5 Tesla brain MRI was performed and $\mathrm{T} 1 \mathrm{w}, \mathrm{T} 2 \mathrm{w}, \mathrm{T} 2$ flair and diffuse axial sections were obtained. MR imaging of the brain revealed lesions with no limited diffusion in the pons central, bilateral cerebellar hemispheres and cerebellar peduncles, deep white matter in the cerebral hemispheres, and in corticalsubcortical region. In addition, the lesions observed in the right half of the mesencephalon and bilateral thalami showed post-contrast staining in the form of spotted or patched points and/or curves (Figures 1-8). No lesions were observed in cervical and thoracic medulla spinalis. Cerebral arterial and venous angiograms were also normal.

\begin{tabular}{|c|c|c|}
\hline & Examinations Performed & Results \\
\hline CSF & Brucella & Negative \\
\hline & Aggl (With Coombs) & Negative \\
\hline & Syphilis IHA & $60 / \mathrm{mm} 3$ \\
\hline CSF Cell & Erythrocyte & $0 / \mathrm{m} 3$ \\
\hline & Leukocyte & Negative \\
\hline & Pandy & No leukocytes were observed \\
\hline & Microscopic examination with direct giemsa staining & No leukocytes or bacteria are observed \\
\hline & Microscopic examination with gram staining & mg/dl \\
\hline CSF & Glucose & . \\
\hline
\end{tabular}




\section{Clinical Radiology \& Imaging Journal}

\begin{tabular}{|c|c|c|}
\hline & Protein & $37 \mathrm{mg} / \mathrm{dl}$ \\
\hline & Chloride & $126 \mathrm{mg} / \mathrm{dl}$ \\
\hline CRP & $0.31 \mathrm{mg} / \mathrm{dl}$ & $<0.5 \mathrm{mg} / \mathrm{dl}$ \\
\hline \multirow[t]{31}{*}{ BLOOD } & Rose-Bengal & Negative \\
\hline & Brucella Agglutination & Negative \\
\hline & Paul-Bannel & Negative \\
\hline & RPR-VDRL & Negative \\
\hline & Syphilis IHA & Negative \\
\hline & ANA, Anti-nDNA & Negative \\
\hline & p-ANCA, c-ANCA & Negative \\
\hline & MPO, PR3 & Negative \\
\hline & Anti-Cardiolipin IgG & Negative \\
\hline & HBsAg, Anti-HBs, & Negative \\
\hline & Toxo IgM, & Negative \\
\hline & Rubella IgM, & Negative \\
\hline & CMV IgM, & Negative \\
\hline & Herpes IgM & Negative \\
\hline & $\mathrm{HIV} \mathrm{Ag/Ab}$ & Negative \\
\hline & Herpes Ig G & Positive \\
\hline & Parvovirus IgG & Positive \\
\hline & Parvovirus IgG & Negative \\
\hline & EBV IG G(Immunoblot) & Positive \\
\hline & Viral capsid Ag(VCA) IgG & \\
\hline & EBNA IG G & Positive \\
\hline & P22 Ig G & Positive \\
\hline & EA IgG & Positive \\
\hline & EBV IG M(Immunoblot) & Negative \\
\hline & (VCA) IgM & \\
\hline & EBNA IgM & Negative \\
\hline & P22 IgM & Negative \\
\hline & EA IgM & Negative \\
\hline & Lyme IgG & Negative \\
\hline & Lyme IgM & Borderline \\
\hline & Anti-CCP & $<0.5 \mathrm{U} / \mathrm{mL}$ \\
\hline
\end{tabular}

Table 1: Examinations performed to 34 year old male patient.

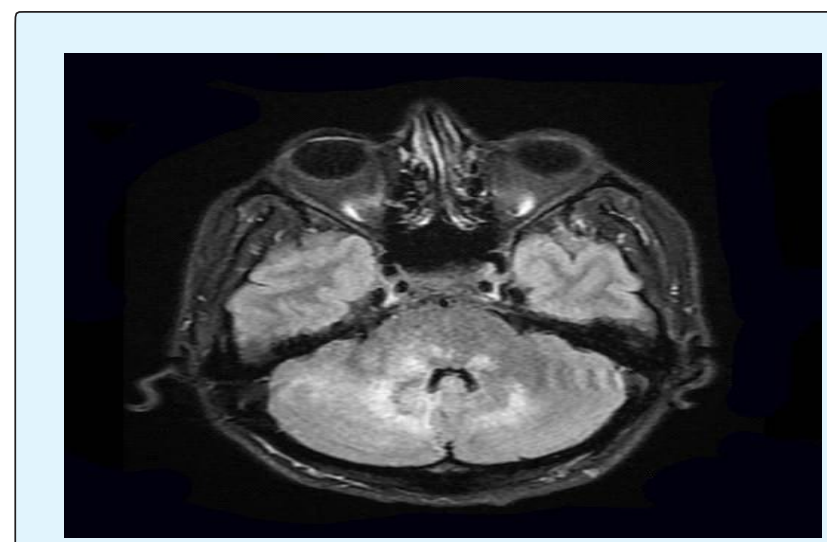

Figure 1: Hiperintens lesions at axial "flair" brain MR images.

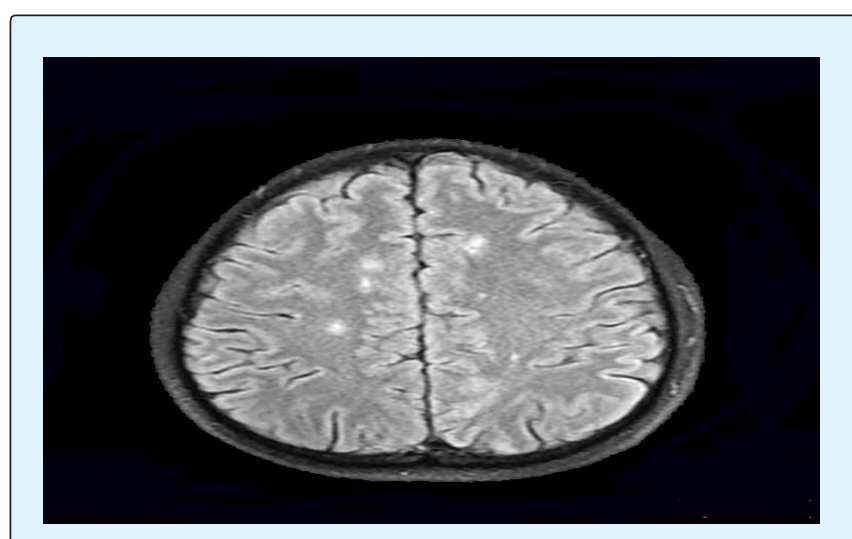

Figure 2: Hiperintens lesions at axial "flair" brain MR images. 


\section{Clinical Radiology \& Imaging Journal}

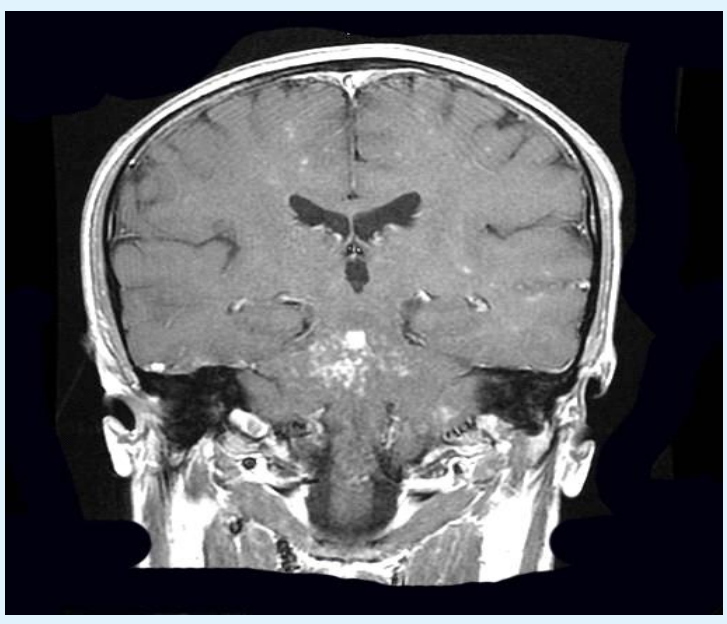

Figure 3: Typical contrast agent uptake in pons, at T1W coronal images.

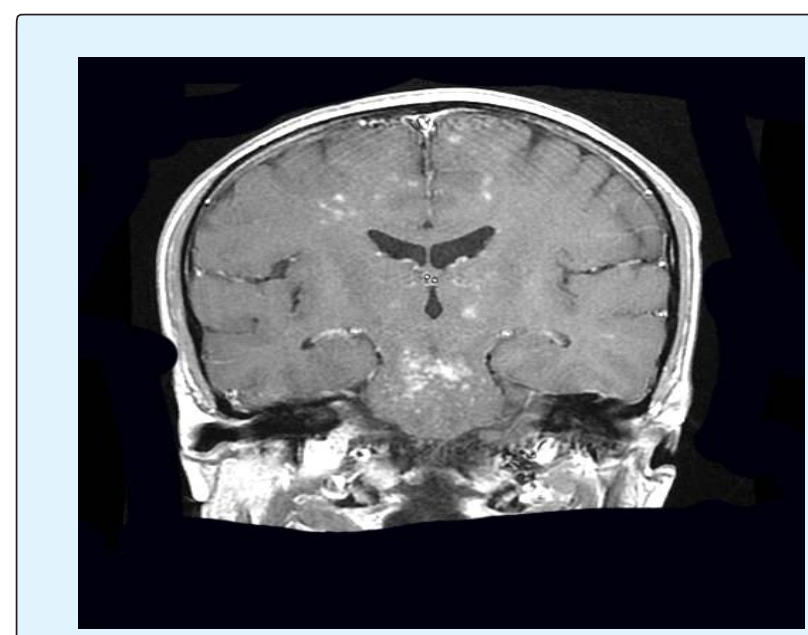

Figure 4: Typical contrast agent uptake in pons, at T1W coronal images.

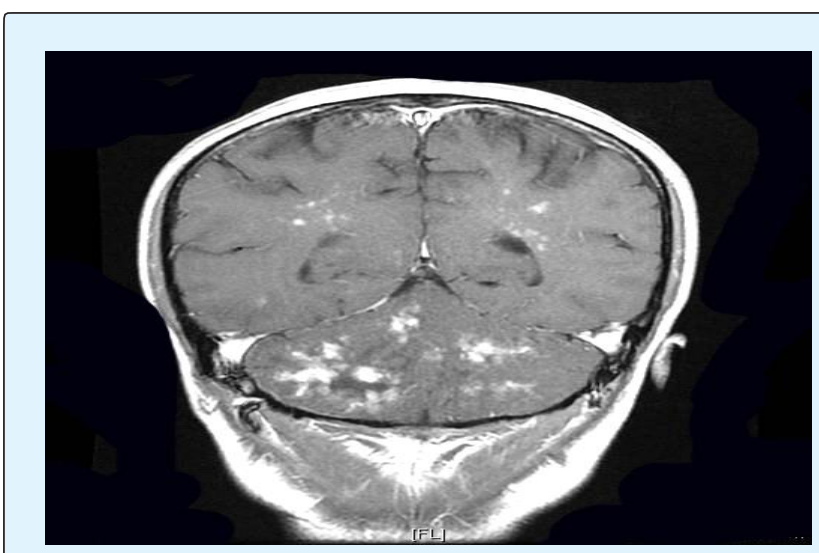

Figure 5: Typical contrast agent uptake in pons and cerebellum (Axial MR images).

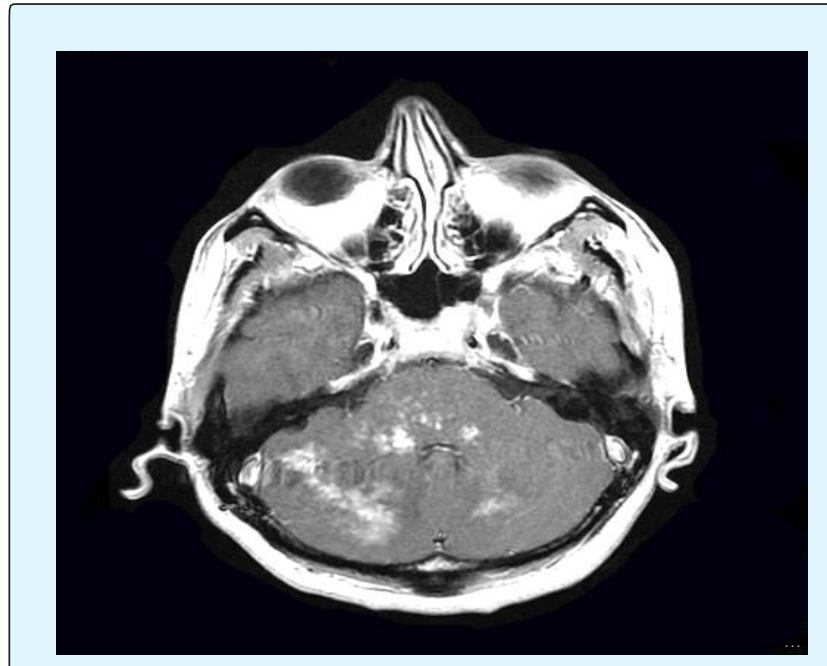

Figure 6: Typical contrast agent uptake in pons and cerebellum (Axial MR images).

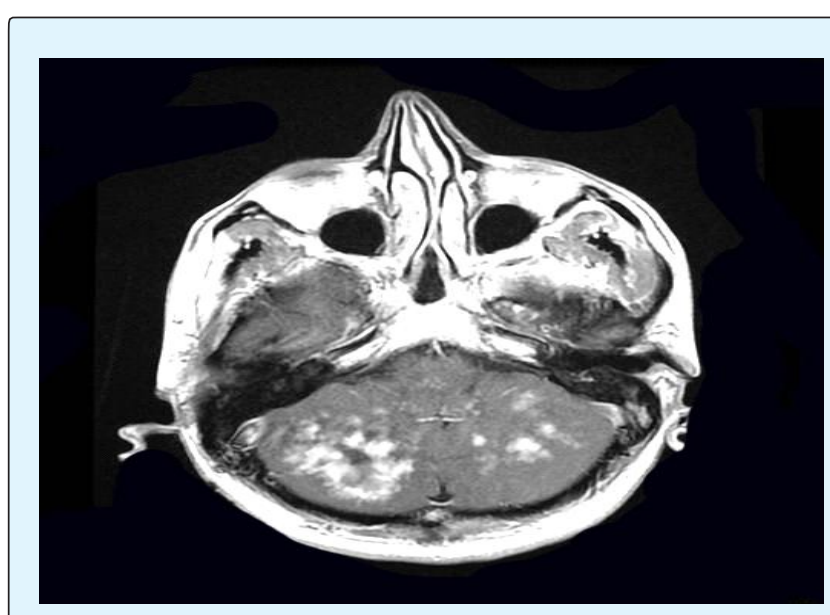

Figure 7: Typical contrast agent uptake in pons and cerebellum at T1W axial images.

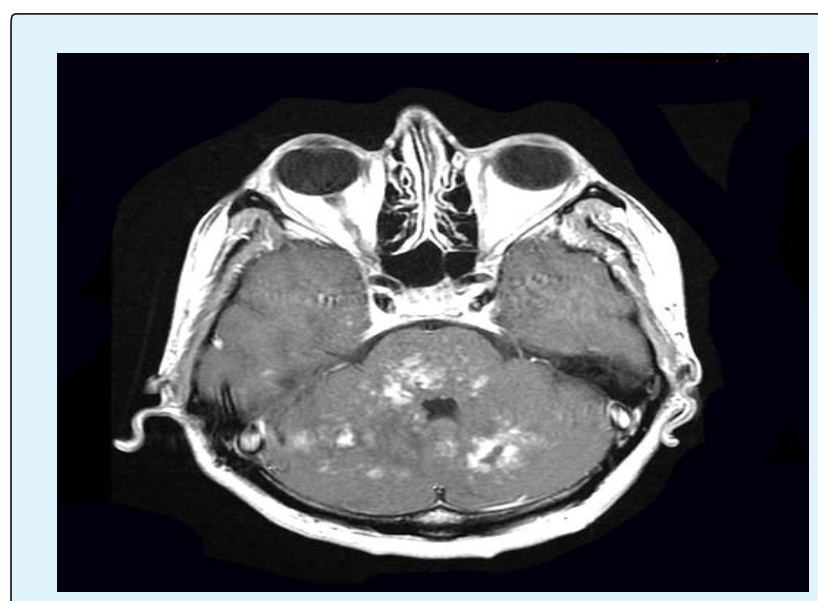

Figure 8: Typical contrast agent uptake in pons and cerebellum at $\mathrm{T} 1 \mathrm{~W}$ axial images. 


\section{Clinical Radiology \& Imaging Journal}

\section{Discussion}

Diagnostic criteria for CLIPPERS have not yet been determined. CLIPPERS diagnosis is based on clinical and neuroimaging findings, and differential diagnosis must be excluded. Subacute and progressive brain stem and cerebellar findings show characteristic MR features. Contrast uptake in the form of spotted curvilinear postcontrast pepper sprinkled appearance is the differential feature of CLIPPERS syndrome. Clinic and MR features of our case were compatible with CLIPPERS. Diagnostic tests for infections, autoimmune diseases and paraneoplastic syndrome were negative. "Bickerstaff" brainstem encephalitis (BBE) was considered in the differential diagnosis. However, in BBE, lesions are usually limited to brainstem. Thalamus and cerebellum are preserved. Other than that, pepper sprinkled like gadolinium uptake involvement has not been reported in BBE. Subacute vascular lesions are excluded because MR angio results are normal. Since serum sodium levels are normal, central pontin myelinolysis is not considered. With clinical and radiological findings, autoimmune encephalitis has also been excluded because lesions' regional anatomy is typical. Sarcoidosis was not considered due to normal lung CT examination. Multiple sclerosis diagnosis is excluded due to absence of typical attacks and according to MR lesions [1-4].

Pathogenesis of CLIPPERS is not known. In most of the patients the apparent cause is not defined. Hillesheim et al. reported a CLIPPERS case following influenza vaccination. Ortega et al. have defined shortly after discontinuation of natalizumab treatment in a case of with MS diganosis. Mashima et al. reported a CLIPPERS case that developed after "Hodgkin lymphoma" treatment. Wang, et al. reported a CLIPPERS case following Herpes Zoster infection.

The main characteristics of CLIPPERS include six (6) criteria. (1) Signs and symptoms of brainstem (2) Gadolinium-bearing lesions in the form of spotted curves; especially involving pons and medial cerebellar peduncles (3) Steroid sensitivity with possible clinical and radiological sequelae (4) Absence of alternative diagnosis (5) Relapsing-remitting course in the absence of immunosuppressive treatment with relapses meeting the first four criteria (6) Lymphohistiocytic infiltrates which are sometimes observed on vessel walls and around vessel in the brainstem biopsy. The interesting and unexplained feature of CLIPPERS is why there is perivascular inflammation that is adjacent to the posterior brain areas and primarily involving the pons. In order to explain this particular characteristic of
CLIPPERS, Pittock, et al. and other authors have discussed a specific immune-mediated process directed against autoantigenic determinants in perivascular regions. These immunological targets may preferably be microstructures located in the pons and peripontin regions. However, these structures have not been defined until now. In this rare case, pathogenetic concepts, in principle, are still based on assumptions and speculations. With more systematic studies, we have to focus on more clearly defining the serologic, radiological and immunopathological markers that exclude this disease from other inflammatory CNS (central nervous system) diseases [5-8].

In summary, CLIPPERS is an immune-mediated inflammatory disease of the brainstem and is reported daily. Irreversible neuronal damage may occur in CLIPPERS. Thus, early use of high-dose intravenous methylprednisolone is necessary. As a result, in our patient, CLIPPERS is diagnosed according to clinical markers and typical radiological findings. The answer to corticosteroids is insufficient in our case. This insufficiency may be due to the failure of early application of corticotherapy and severity of inflammation due to diffuse radiological findings.

\section{References}

1. Zalewski NL, Tobin WO (2017) CLIPPERS. Curr Neurol Neurosci Rep 17(9): 65.

2. Hou X, Wang X, Xie BO, Lin W, Liu J, et al. (2016) Horizontal eyeball akinesia as an initial manifestation of CLIPPERS Case report and review of literature . Medicine 95(34): 4640.

3. Taieb G, Duran-Peña A, de Chamfleur NM, Moulignier A, Thouvenot E, et al. (2016) Punctate and curvilinear gadolinium enhancing lesions in the brain: a practical approach. Neuroradiol 58(3): 221-235.

4. Dudesek A, Rimmele F, Tesar S, Kolbaske S, Rommer PS, et al. (2014) CLIPPERS: chronic lymphocytic inflammation with pontine perivascular enhancement responsive to steroids. Review of an increasingly recognized entity within the spectrum of inflammatory central nervous system disorders. Clinic Exp Immunol 175(3): 385-396.

5. Simon NG, Parratt JD, Barnett MH, Bucland ME, Gupta $\mathrm{R}$, et al. (2012) Expanding the clinical, radiological and neuropathological phenotype of chronic lymphocytic inflammation with pontine perivascular 


\section{Clinical Radiology \& Imaging Journal}

enhancement responsive to steroids CLIPPERS.J Neurol Neusurg Psychiatry 83(1): 15-22.

6. Wang X, Huang D, Huang X, Zhang J, Ran Y, et al. (2017) Chronic lymphocytic inflammation with pontine perivascular enhancement responsive to steroids (CLIPPERS): A lymphocytic reactive response of the central nervous system? A case report. J of Neuroimmunol 305: 68-71.
7. Mubasher M, Sukik A, Hassan El BA, Rahil A (2017) Chronic Lymphocytic Inflammation with Pontine Perivascular Enhancement Responsive to Steroids, with Cranial and Caudal Extension. Case Reports in Neurological Medicine 2017: 1-4.

8. Taieb G, Allou T, Labauge P (2017) Therapeutic Approaches in CLIPPERS. Curr Treat Options Neurol 19(5): 17. 